\title{
Low diffusion E-CUSP scheme with implicit high order WENO scheme for preconditioned Navier-Stokes equations
}

\author{
Yiqing Shen ${ }^{\mathrm{a}}$, Gecheng Zha ${ }^{\mathrm{b}, *}$ \\ ${ }^{a}$ LHD, Institute of Mechanics, Chinese Academy of Sciences, Beijing 100190, China \\ ${ }^{\mathrm{b}}$ Dept. of Mechanical and Aerospace Engineering, Miami Wind ${ }^{\mathrm{TM}}$, University of Miami, Coral Gables, Florida 33124, United States
}

\section{A R T I C L E I N F O}

\section{Article history:}

Received 22 October 2010

Received in revised form 21 August 2011

Accepted 23 October 2011

Available online 4 November 2011

\section{Keywords:}

Preconditioing

WENO scheme

All flow speeds

Incompressible flows

Compressible flows

\begin{abstract}
A B S T R A C T
A low diffusion E-CUSP (LDE) scheme for preconditioned Navier-Stokes equations is developed. The LDE scheme with high-order WENO reconstruction and high order difference scheme for viscous terms is used to simulate several flows at various speed from low speed natural convection to supersonic flows. The unfactored implicit Gauss-Seidel relaxation scheme, in which the preconditioned Roe's matrices are used, is used for time integration. Numerical results are presented to show efficiency, accuracy and robustness of the new preconditioning scheme.
\end{abstract}

(c) 2011 Elsevier Ltd. All rights reserved.

\section{Introduction}

In recent years, the convective upwind and split pressure (CUSP) family schemes have achieved great success. The CUSP schemes can be basically categorized to two types, the H-CUSP and E-CUSP [1-3]. The H-CUSP schemes have the total enthalpy from the energy equation in their convective vector, while the ECUSP schemes use the total energy in the convective vector. The Liou's AUSM family schemes [4-9], van Leer-Hänel scheme [10], and Edwards's LDFSS schemes [11,12] belong to the H-CUSP group. The schemes developed by Zha et al. [13-17] belong to the E-CUSP group.

From the characteristic theory point of view, the H-CUSP schemes are not fully consistent with the disturbance propagation directions $[18,19]$, which may affect the stability and robustness of the schemes. By splitting the eigenvalues of the Jacobians to convection (velocity) and waves (speed of sound), one will find that the convection terms only contain the total energy [13], which will lead to the E-CUSP schemes.

With the application of computational fluid dynamics becoming more and more popular, the demand for developing a unified algorithm for compressible and incompressible flows becomes stronger to satisfy the needs of broad engineering problems. When direct applying compressible flow equation to incompressible flows, there exist two problems: stiffness and large numerical dissipation.

\footnotetext{
* Corresponding author.

E-mail addresses: yqshen@imech.ac.cn (Y. Shen), gzha@miami.edu (G. Zha).
}

Both problems are due to a large difference between the speed of sound and flow speed. The large dissipation may cause low convergence, distort the solution of a wall boundary layer, etc.

Preconditioning is to change the eigenvalues of the compressible flow equations system in order to remove the large disparity of wave speeds. Usually, the system of compressible flow equations is preconditioned by multiplying the time derivatives with a proper matrix [20-24]. Thornber et al. [25,26] propose an interesting method by modifying the variable reconstruction to allow minimum dissipation of low Mach number features whilst maintaining shock capturing ability, all without changing the formulation of the governing equations.

For the spatial discretization with preconditioning, the 2nd order central differencing is adopted by Choi and Merkle [23,27] and De Bortoli [28]. The Roe-type flux-difference splitting (FDS) is used by Weiss and Smith [24,29-32]. The 3rd-order MUSCL extrapolation is used by Briley et al. [33]. The flux-vector splitting (FVS) is applied by Turkle et al. [34]. Patel and Drikakis [35] investigate the effects of preconditioning on the accuracy and efficiency of separated flows. Edwards and his colleagues [36-39] have extended their H-CUSP schemes to all flow speeds. There is no work to extend E-CUSP schemes to preconditioning.

The purpose of this paper is to develop a preconditioned low diffusion E-CUSP scheme with high order WENO scheme for Navier-Stokes equations at all flow speeds. With unfactored implicit Gauss-Seidel relaxation scheme for time integration, the LDE scheme is used to simulate various flow fields at all speeds. The numerical solutions of a lid driven cavity flow, a natural 
convective cavity flow, subsonic flow, transonic and supersonic flows show that the suggested preconditioning method with high-order schemes is efficient, robust, and accurate for both low speed incompressible flows and high speed compressible flows.

\section{Numerical method}

\subsection{Governing equations}

The preconditioned system for steady state flows in generalized coordinate is obtained by multiplying the preconditioning matrix $\Gamma$ to the time derivative terms of Navier-Stokes equations to give

$\Gamma \frac{\partial q}{\partial t}+\frac{\partial E}{\partial \xi}+\frac{\partial F}{\partial \eta}+\frac{\partial G}{\partial \zeta}=\frac{1}{R e}\left(\frac{\partial R}{\partial \xi}+\frac{\partial S}{\partial \eta}+\frac{\partial T}{\partial \zeta}\right)$

The preconditioning matrix $\Gamma$ has various forms [20,22-24], and is dependent on the choice of $q$. This paper adopts the method of Weiss and Smith described in Ref. [24]. The $q$ and $\Gamma$ are taken as the following,

$q=(p, u, v, w, T)^{T}$

$\Gamma=\left[\begin{array}{ccccc}\Theta & 0 & 0 & 0 & \rho_{T} \\ \Theta u & \rho & 0 & 0 & \rho_{T} u \\ \Theta v & 0 & \rho & 0 & \rho_{T} v \\ \Theta w & 0 & 0 & \rho & \rho_{T} w \\ \Theta H-1 & \rho u & \rho v & \rho w & \rho_{T} H+\rho C_{p}\end{array}\right]$

where $\Theta$ is given by

$\Theta=\left(\frac{1}{U_{r}^{2}}-\frac{\rho_{T}}{\rho C_{p}}\right)$

$U_{r}$ is a reference velocity. In this paper, the reference velocity proposed by Edwards and Roy [36] is used:

$U_{r}=\min \left[c, \max \left(|V|, k\left|V_{\infty}\right|\right)\right]$

where $c$ is the speed of sound, $|V|=\sqrt{u^{2}+v^{2}+w^{2}}$ is the velocity magnitude, $\left|V_{\infty}\right|$ is a reference velocity. $H$ is the total enthalpy, $\rho_{T}$ stands for $\frac{\partial \rho}{\partial T}, C_{p}$ is the specific heat at constant pressure. $k$ is a constant [31]. For all case calculated in this paper, $U_{r}=1$ and $k=0.5$ are used.

The eigenvalues of Jacobian matrix $\Gamma^{-1} \frac{\partial E}{\partial q}$ are

$U, U, U, U^{\prime}+C^{\prime}, U^{\prime}-C^{\prime}$

where

$U=l_{x} u+l_{y} v+l_{z} w$

$U^{\prime}=\frac{1}{2} U\left(1+M_{r}^{2}\right), \quad C^{\prime}=\frac{1}{2} \sqrt{\left(1-M_{r}^{2}\right) U^{2}+4 M_{r}^{2} C^{2}}$

and

$M_{r}=\frac{U_{r}}{c}, \quad C=c \sqrt{l_{x}^{2}+l_{y}^{2}+l_{z}^{2}}$

$l$ is the normal vector on $\xi$ surfaces with its magnitude equal to the elemental surface area and pointing to the direction of increasing $\xi$.

$\mathbf{l}=l_{x} i+l_{y} j+l_{z} k=\frac{\nabla \xi}{J} \mathrm{~d} \eta \mathrm{d} \zeta$

$J$ is the transformation Jacobian. The eigenvalues given in Eq. (2) will have the same order of magnitude when the velocity approaches zero. This is the purpose of preconditioning to improve the condition number of the Jacobian and remove the stiffness.

\subsection{Preconditioning of the low diffusion E-CUSP (LDE) scheme}

The preconditioning methodology of this paper is based on the recent E-CUSP scheme suggested by Zha et al. [17]. In $[13,14,16,17]$, the characteristic analysis is given as the foundation to construct the E-CUSP scheme. The basic idea is to split the flux $\mathbf{E}$ to the convective flux $\mathbf{E}^{\mathbf{c}}$ and the pressure flux $\mathbf{E}^{\mathbf{p}}$. That is:

$\mathbf{E}=E^{c}+E^{p}=\left(\begin{array}{c}\rho U \\ \rho u U \\ \rho v U \\ \rho w U \\ \rho e U\end{array}\right)+\left(\begin{array}{c}0 \\ l_{x} p \\ l_{y} p \\ l_{z} p \\ p U\end{array}\right)$

The flux at interface $\frac{1}{2}, E_{1 / 2}$ is evaluated as,

$E_{1 / 2}=E_{1 / 2}^{c}+E_{1 / 2}^{p}$

where

$E_{1 / 2}^{c}=U^{+} f_{L}^{c}+U^{-} f_{R}^{c}$

and

$E_{\frac{1}{2}}^{p}=p_{1 / 2}\left(\begin{array}{c}0 \\ l_{x} \\ l_{y} \\ l_{z} \\ U_{1 / 2}\end{array}\right), \quad f^{c}=\left(\begin{array}{c}\rho \\ \rho u \\ \rho v \\ \rho w \\ \rho e\end{array}\right)$

$p_{1 / 2}=P^{+} p_{L}+P^{-} p_{R}$

The different formulations for $U^{+}, U^{-}, P^{+}$and $P^{-}$can be found in $[13,14,16,17]$ with different behavior.

The preconditioning of the LDE scheme needs to satisfy two conditions when the flow velocity approaching zero: (1) the eigenvalues of the Jacobian matrices should be at the same order of magnitude of the velocity and (2) the numerical dissipation should diminish. The condition 1 is explained in the previous section. The condition 2 is described as the following.

The interface flux $E_{1 / 2}$ can be generally expressed as consisting of a central differencing plus a numerical dissipation $\mathbf{D}$ as [36]

$E_{1 / 2}=\frac{1}{2}\left[E_{L}+E_{R}+\mathbf{D}(L, R)\right]$

Similarly, the interface pressure $p_{1 / 2}$ of (8) can be written as [38]

$$
\begin{aligned}
p_{1 / 2} & =P^{+} p_{L}+P^{-} p_{R} \\
& =\frac{1}{2}\left[\left(p_{L}+p_{R}\right)+\left(P^{+}-P^{-}\right)\left(p_{L}-p_{R}\right)+\left(P^{+}+P^{-}-1\right)\left(p_{L}+p_{R}\right)\right]
\end{aligned}
$$

The last two terms can be regarded as the diffusion terms of $p_{1 / 2}$. A low Mach number results in large pressure value, hence the second diffusion term could be excessively large. A better scaling is found by replacing $\left(p_{L}+p_{R}\right)$ by $2 \rho_{1 / 2} \tilde{c}_{1 / 2}^{2}$ or $2 \rho_{1 / 2} U_{r, 1 / 2}^{2}[37,38]$. More comprehensive analysis can be found in Ref. [40] for single-phase flows and in Ref. [41] for two-phase flows. Hence, Eq. (10) is replaced by

$$
\begin{aligned}
p_{1 / 2}= & P^{+} p_{L}+P^{-} p_{R}=\frac{1}{2}\left[\left(p_{L}+p_{R}\right)+\left(P^{+}-P^{-}\right)\left(p_{L}-p_{R}\right)\right. \\
& \left.+\rho_{1 / 2} U_{r, 1 / 2}^{2}\left(P^{+}+P^{-}-1\right)\right]
\end{aligned}
$$

Similarly, the split velocities $U^{ \pm}$used in the low-diffusion flux splitting scheme can be written as

$$
\begin{aligned}
& U^{+}=\tilde{c}_{1 / 2}\left[M^{+}-M_{1 / 2}^{+}\right] \\
& U^{-}=\tilde{c}_{1 / 2}\left[M^{-}+M_{1 / 2}^{-}\right]
\end{aligned}
$$


where

$M_{1 / 2}^{+}=M_{1 / 2}\left(1-\frac{p_{L}-p_{R}+\delta\left|p_{L}-p_{R}\right|}{p_{L}+p_{R}}\right)$
$M_{1 / 2}^{-}=M_{1 / 2}\left(1+\frac{p_{L}-p_{R}-\delta\left|p_{L}-p_{R}\right|}{p_{L}+p_{R}}\right)$

Using $2 \rho_{L} U_{r, 1 / 2}^{2}$ and $2 \rho_{R} U_{r, 1 / 2}^{2}$ to replace $\left(p_{L}+p_{R}\right)$ in Eqs. (12) and (13), respectively, the new split velocities are obtained as

$U^{+}=\tilde{c}_{1 / 2}\left[M^{+}-M_{1 / 2}\left(1-\frac{p_{L}-p_{R}+\delta\left|p_{L}-p_{R}\right|}{2 \rho_{L} U_{r, 1 / 2}^{2}}\right)\right]$
$U^{-}=\tilde{c}_{1 / 2}\left[M^{-}+M_{1 / 2}\left(1+\frac{p_{L}-p_{R}-\delta\left|p_{L}-p_{R}\right|}{2 \rho_{R} U_{r, 1 / 2}^{2}}\right)\right]$

$\delta=1$ is used in [38], $\delta=0$ is used in [39] and also in this paper. Other quantities used in the preceding definitions are

$$
\begin{aligned}
& M^{+}=\alpha_{L}^{+}\left(1+\beta_{L}\right) M_{L}-\beta_{L} M_{L}^{+} \\
& M^{-}=\alpha_{R}^{-}\left(1+\beta_{R}\right) M_{R}-\beta_{R} M_{R}^{-} \\
& M_{L, R}^{ \pm}= \pm \frac{1}{4}\left(M_{L, R} \pm 1\right)^{2} \\
& \alpha_{L, R}^{ \pm}=\frac{1}{2}\left[1 \pm \operatorname{sign}\left(1.0, M_{L, R}\right)\right] \\
& \beta_{L, R}=-\max \left[0,1-\operatorname{int}\left(\left|M_{L, R}\right|\right)\right] \\
& M_{1 / 2}=\frac{1}{2}\left(M^{+}-\alpha_{L}^{+} M_{L}-M^{-}+\alpha_{R}^{-} M_{R}\right) \\
& P^{ \pm}=\alpha_{L, R}^{ \pm}\left(1+\beta_{L, R}\right)-\frac{\beta_{L, R}}{2}\left[1 \pm M_{L, R}\right] \\
& \tilde{c}_{1 / 2}=\left.\frac{\sqrt{\left(1-M_{r}^{2}\right)^{2} U^{2}+4 C^{2} M_{r}^{2}}}{1+M_{r}^{2}}\right|_{1 / 2}
\end{aligned}
$$

and

$M_{L, R}=\frac{U_{L, R}}{\tilde{c}_{1 / 2}}$

Since the H-CUSP scheme uses total enthalpy and the E-CUSP scheme uses total energy in Eq. (4) for the convective vector, $\mathrm{H}$ CUSP schemes $[36,38,39]$ hence do not have the term of $p_{1 / 2} U_{1 / 2}$ in Eq. (7). In this paper, the interface velocity $U_{1 / 2}$ is evaluated as

$U_{1 / 2}=U^{+}+U^{-}$

\subsection{The WENO reconstruction}

The high order accuracy of $E_{i+1 / 2}$ is obtained by achieving the high order accuracy of the left and right primitive variables $q^{L}$ and $q^{R}$ using the WENO scheme described below. This procedure is similar to the MUSCL scheme suggested by van Leer [42] and is adopted in [43].

The finite difference 5th-order accuracy WENO scheme suggested by Jiang and Shu [44] is used to evaluate the primitive variables $q^{L}$ and $q^{R}$. The WENO scheme for variable $q^{L}$ can be written as:

$q_{i+1 / 2}^{L}=\omega_{0} q_{0}+\omega_{1} q_{1}+\omega_{2} q_{2}$

where $\omega_{0}, \omega_{1}$ and $\omega_{2}$ are the weights, and the $q_{0}, q_{1}$ and $q_{2}$ are the 3rd order accuracy reconstruction of the variables in three different stencils. They are determined as the following

$\left\{\begin{array}{l}q_{0}=\frac{1}{3} q_{i-2}-\frac{7}{6} q_{i-1}+\frac{11}{6} q_{i} \\ q_{1}=-\frac{1}{6} q_{i-1}+\frac{5}{6} q_{i}+\frac{1}{3} q_{i+1} \\ q_{2}=\frac{1}{3} q_{i}+\frac{5}{6} q_{i+1}-\frac{1}{6} q_{i+2}\end{array}\right.$

$$
\begin{aligned}
& \omega_{k}=\frac{\alpha_{k}}{\alpha_{0}+\cdots+\alpha_{r-1}}, \\
& \alpha_{k}=\frac{C_{k}}{\left(\varepsilon+I S_{k}\right)^{p}}, \quad k=0,1,2
\end{aligned}
$$

where $C_{k}$ are the optimal weights with the following values:

$C_{0}=0.1, \quad C_{1}=0.6, \quad C_{2}=0.3$

The $I S_{k}$ are the smooth estimators determined as

$$
\left\{\begin{array}{l}
I S_{0}=\frac{13}{12}\left(q_{i-2}-2 q_{i-1}+q_{i}\right)^{2}+\frac{1}{4}\left(q_{i-2}-4 q_{i-1}+3 q_{i}\right)^{2} \\
I S_{1}=\frac{13}{12}\left(q_{i-1}-2 q_{i}+q_{i+1}\right)^{2}+\frac{1}{4}\left(q_{i-1}-q_{i+1}\right)^{2} \\
I S_{2}=\frac{13}{12}\left(q_{i}-2 q_{i+1}+q_{i+2}\right)^{2}+\frac{1}{4}\left(3 q_{i}-4 q_{i+1}+q_{i+2}\right)^{2}
\end{array}\right.
$$

The $\varepsilon$ in Eq. (20) is introduced to avoid the denominator becoming zero. Jiang and Shu's numerical tests indicate that the results are not sensitive to the choice of $\varepsilon$ as long as it is in the range of $10^{-5}$ $10^{-7}$. In their paper [44], $\varepsilon$ is taken as $10^{-6}$. In Ref. [43], the $\varepsilon$ value of $10^{-2}$ suggested by Shen et al. to suppress the oscillation of $I S_{k}$ and improve the convergence and accuracy is adopted in this paper.

The $q^{R}$ is constructed symmetrically as $q^{L}$ about $i+1 / 2$.

\subsection{The 4th-order schemes for viscous terms [45]}

A set of fully conservative 4th-order accurate finite central differencing schemes using the same stencil width of the WENO scheme for the viscous terms is used in this paper [45]. The scheme for the viscous derivative term $\frac{\partial R}{\partial \xi}$ in Navier-Stokes equations Eq. (1) can be written as the following,

$\left.\frac{\partial R}{\partial \xi}\right|_{i}=\frac{\widetilde{R}_{i+1 / 2}-\widetilde{R}_{i-1 / 2}}{\Delta \xi}$

To obtain 4 th order accuracy, $\widetilde{R}$ needs to be reconstructed as

$\widetilde{R}_{i-1 / 2}=\sum_{I=i-3 / 2}^{i+1 / 2} \alpha_{I} R_{I}$

where

$\alpha_{i-3 / 2}=-\frac{1}{24}, \quad \alpha_{i-1 / 2}=\frac{26}{24}, \quad \alpha_{i+3 / 2}=-\frac{1}{24}$

$R_{i-1 / 2}=\left[\left(\xi_{x} \tau_{x x}\right)+\left(\eta_{y} \tau_{x y}\right)+\left(\zeta_{z} \tau_{x z}\right)\right]_{i-1 / 2}$

$$
\begin{aligned}
\left(\tau_{x x}\right)= & \mu\left\{\frac{4}{3}\left[\left(\xi_{x} \frac{\partial u}{\partial \xi}\right)+\left(\eta_{x} \frac{\partial u}{\partial \eta}\right)+\left(\zeta_{x} \frac{\partial u}{\partial \zeta}\right)\right]-\frac{2}{3}\left[\left(\xi_{y} \frac{\partial v}{\partial \xi}\right)\right.\right. \\
& \left.\left.+\left(\eta_{y} \frac{\partial v}{\partial \eta}\right)+\left(\zeta_{y} \frac{\partial v}{\partial \zeta}\right)\left(\xi_{z} \frac{\partial w}{\partial \xi}\right)+\left(\eta_{z} \frac{\partial w}{\partial \eta}\right)+\left(\zeta_{z} \frac{\partial w}{\partial \zeta}\right)\right]\right\}
\end{aligned}
$$

If $R_{I}$ in Eq. (23) can be approximated with the accuracy order not lower than 4th order, the Taylor expansion analysis of (22) and (23) will give the following relation [45]

$\frac{1}{\Delta \xi}\left(\widetilde{R}_{i+1 / 2}-\widetilde{R}_{i-1 / 2}\right)=R^{\prime}\left(\xi_{i}\right)+O\left(\Delta \xi^{4}\right)$

i.e. the 4th order accuracy is achieved.

In order to achieve the highest order accuracy of $R_{I}$ with $I=i-$ $3 / 2, i-1 / 2, i+1 / 2$, the approximation of each component in Eq. (23) using all the involved points of the WENO stencil is given below:

$\left\{\begin{array}{l}\mu_{I}=\sum_{l=m}^{n} C_{l}^{I} \mu_{i+l}, \\ \left.\frac{\partial u}{\partial \xi}\right|_{I}=\frac{1}{\Delta \xi} \sum_{l=r}^{s} D_{l}^{I} u_{i+l}, \\ \left.\frac{\partial u}{\partial \eta}\right|_{I}=\left.\sum_{l=m}^{n} C_{l}^{I} \frac{\partial u}{\partial \eta}\right|_{i+l, j}\end{array}\right.$ 
Table 1

The coefficients of $C_{l}^{I}$.

\begin{tabular}{lllll}
\hline $\mathrm{I}$ & $C_{-2}^{I}$ & $C_{-1}^{I}$ & $C_{0}^{I}$ & $C_{1}^{I}$ \\
\hline$i-3 / 2$ & $5 / 16$ & $15 / 16$ & $-5 / 16$ & $1 / 16$ \\
$i-1 / 2$ & $-1 / 16$ & $9 / 16$ & $9 / 16$ & $-1 / 16$ \\
$i+1 / 2$ & $1 / 16$ & $-5 / 16$ & $15 / 16$ & $5 / 16$ \\
\hline
\end{tabular}

where

$\left.\frac{\partial u}{\partial \eta}\right|_{i, j}=\frac{1}{\Delta \eta} \sum_{l=p}^{q} C_{l}^{c} u_{i, j+l}$

By choosing different ranges for $(m, n),(r, s),(p, q)$ and different coefficients $C_{l}^{l}, D_{l}^{I}, C_{l}^{c}$, one can obtain different order accuracy approximation to the viscous terms. The principle of choosing $(m, n),(r, s),(p, q)$ is to ensure that the approximation of $\left.\frac{\partial R}{\partial \xi}\right|_{i}$ in Eq. $(22)$ is a central differencing. For example, in this paper, $(m, n)=(-2,1),(r, s)=(-3,2)$, and $(p, q)=(-2,2)$ are used, and they give [45]

$$
\left\{\begin{array}{l}
\mu_{I}=\sum_{l=m}^{n} C_{l}^{I} \mu_{i+l}+O\left(\Delta \xi^{4}\right) \\
\left.\frac{\partial u}{\partial \xi}\right|_{I}=\frac{1}{\Delta \xi} \sum_{l=r}^{s} D_{l}^{I} u_{i+l}+O\left(\Delta \xi^{5}\right) \\
\left.\frac{\partial u}{\partial \eta}\right|_{I}=\left.\sum_{l=m}^{n} C_{l}^{I} \frac{\partial u}{\partial \eta}\right|_{i+l, j}+O\left(\Delta \xi^{4}, \Delta \eta^{4}\right)
\end{array}\right.
$$

where

$\left.\frac{\partial u}{\partial \eta}\right|_{i, j}=\frac{1}{\Delta \eta} \sum_{l=p}^{q} C_{l}^{c} u_{i, j+l}+O\left(\Delta \eta^{4}\right)$

the coefficients $C_{l}^{I}, D_{l}^{I}, C_{l}^{c}$ can be obtained by Taylor's series expansion and are given in Tables 1-3.

\section{Time marching method}

The implicit discretized formula of Eq. (1) can be expressed as following

$$
\left(\frac{\Gamma}{\Delta t}+\left.\frac{\partial R H S}{\partial q}\right|^{\prime}\right) \delta q^{n+1}=R H S^{n}
$$

where RHS is the residual calculated by using inviscid numerical fluxes and viscous fluxes, such as Eqs. (5) and (22), $\left.\frac{\partial R H S}{\partial q}\right|^{\prime}$ is the approximation of Jacobian matrix $\frac{\partial R H S}{\partial q}$.

For the preconditioned E-CUSP scheme, a first order discretization is used for solving the corresponding Jacobian matrices on the left hand side of Eq. (30). For example, for convective flux $E_{i+1 / 2}$

Table 2

The coefficients of $D_{l}^{I}$.

\begin{tabular}{lllllll}
\hline $\mathrm{I}$ & $D_{-3}^{I}$ & $D_{-2}^{I}$ & $D_{-1}^{I}$ & $D_{0}^{I}$ & $D_{1}^{I}$ & $D_{2}^{I}$ \\
\hline$i-3 / 2$ & $71 / 1920$ & $-141 / 128$ & $69 / 64$ & $1 / 192$ & $-3 / 128$ & $3 / 640$ \\
$i-1 / 2$ & $-3 / 640$ & $25 / 384$ & $-75 / 64$ & $75 / 64$ & $-25 / 384$ & $3 / 640$ \\
$i+1 / 2$ & $-3 / 640$ & $3 / 128$ & $-1 / 192$ & $-69 / 64$ & $141 / 128$ & $-71 / 1920$
\end{tabular}

Table 3

The coefficients of $C_{l}^{c}$.

\begin{tabular}{lllll}
\hline$C_{-2}^{c}$ & $C_{-1}^{c}$ & $C_{0}^{c}$ & $C_{1}^{c}$ & $C_{2}^{c}$ \\
\hline $1 / 12$ & $-8 / 12$ & 0 & $8 / 12$ & $-1 / 12$ \\
\hline
\end{tabular}

$$
\begin{aligned}
\frac{\partial E_{i+1 / 2}}{\partial q_{i+1 / 2}} \delta q_{i+1 / 2}= & \frac{1}{2}\left[\left.\frac{\partial E}{\partial q}\right|_{i}+\left.\left(\Gamma M_{\Gamma}\left|\Lambda_{\Gamma}\right| M_{\Gamma}^{-1}\right)\right|_{i+1 / 2}\right] \delta q_{i} \\
& +\frac{1}{2}\left[\left.\frac{\partial E}{\partial q}\right|_{i+1}-\left.\left(\Gamma M_{\Gamma}\left|\Lambda_{\Gamma}\right| M_{\Gamma}^{-1}\right)\right|_{i+1 / 2}\right] \delta q_{i+1}
\end{aligned}
$$

where $\Lambda_{\Gamma}$ is the diagonal matrix of eigenvalues Eq. (2), $M_{\Gamma}$ and $M_{\Gamma}^{-1}$ are the matrices of right and left eigenvectors,

$A=\frac{\partial E}{\partial q}=\left[\begin{array}{ccccc}\rho_{p} U & \rho l_{x} & \rho l_{y} & \rho l_{z} & \rho_{T} U \\ \rho_{p} u U+l_{x} & \rho U+\rho u l_{x} & \rho u l_{y} & \rho u l_{z} & \rho_{T} u U \\ \rho_{p} v U+l_{y} & \rho v l_{x} & \rho U+\rho v l_{y} & \rho v l_{z} & \rho_{T} v U \\ \rho_{p} w U+l_{z} & \rho w l_{x} & \rho w l_{y} & \rho U+\rho w l_{z} & \rho_{T} w U \\ \rho_{p} H U & \rho u U+\rho H l_{x} & \rho v U+\rho H l_{y} & \rho w U+\rho H l_{z} & \rho_{T} H U+\rho C_{p} U\end{array}\right]$

$M_{\Gamma}=\left[\begin{array}{ccccc}0 & 0 & 0 & 1 & 1 \\ 0 & m_{x}^{\prime} & n_{x}^{\prime} & \frac{-l_{x}^{\prime}}{X_{1}} & \frac{-l_{x}^{\prime}}{X_{2}} \\ 0 & m_{y}^{\prime} & n_{y}^{\prime} & \frac{-l_{y}^{\prime}}{X_{1}} & \frac{-l_{y}^{\prime}}{X_{2}} \\ 0 & m_{z}^{\prime} & n_{z}^{\prime} & \frac{-l_{z}^{\prime}}{X_{1}} & \frac{-l_{z}^{\prime}}{X_{2}} \\ 1 & 0 & 0 & X_{3} & X_{4}\end{array}\right]$

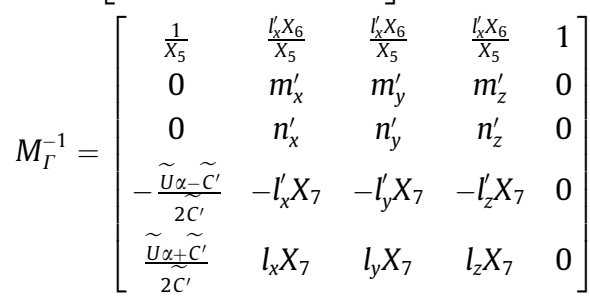

where

$X_{1}=\tilde{\rho}\left(\widetilde{U} \alpha-\widetilde{C}^{\prime}\right), \quad X_{2}=\tilde{\rho}\left(\widetilde{U} \alpha+\widetilde{C}^{\prime}\right)$

$X_{3}=\frac{1-\left(\rho_{p}-\Theta\right) \widetilde{U}\left(\widetilde{U} \alpha-\widetilde{C}^{\prime}\right)}{\left(\rho_{T}+\tilde{\rho} C_{p} \Theta\right)\left(\widetilde{U} \alpha-\widetilde{C}^{\prime}\right)^{2}}, X_{4}=\frac{1-\left(\rho_{p}-\Theta\right) \widetilde{U}\left(\widetilde{U} \alpha+\widetilde{C}^{\prime}\right)}{\left(\rho_{T}+\tilde{\rho} C_{p} \Theta\right)\left(\widetilde{U} \alpha+\widetilde{C}^{\prime}\right)^{2}}$

$X_{5}=\left(\rho_{T}+\tilde{\rho} C_{p} \Theta\right)\left(\widetilde{U}^{2} \alpha^{2}-\widetilde{C}^{\prime 2}\right), X_{6}=\tilde{\rho} \widetilde{U}\left[2 \alpha-\left(\rho_{p}-\Theta\right)\left(\widetilde{U}^{2} \alpha^{2}-\widetilde{C}^{\prime 2}\right)\right]$

$X_{7}=\frac{\tilde{\rho}\left(\widetilde{U}^{2} \alpha^{2}-\widetilde{C}^{\prime 2}\right)}{2 \widetilde{C}^{\prime}}$

and

$\widetilde{U}=l_{x}^{\prime} \tilde{u}+l_{y}^{\prime} \tilde{v}+l_{z}^{\prime} \tilde{w}, \quad \widetilde{C}^{\prime}=\sqrt{\alpha^{2} \widetilde{U}^{2}+U_{r}^{2}}$

where

$\overrightarrow{\mathbf{l}^{\prime}}=\left(l_{x}^{\prime}, l_{y}^{\prime}, l_{z}^{\prime}\right)=\frac{\overrightarrow{\mathbf{l}}}{|\overrightarrow{\mathbf{l}}|}, \quad \overrightarrow{\mathbf{l}^{\prime}} \cdot \overrightarrow{\mathbf{m}}^{\prime}=0, \overrightarrow{\mathbf{l}^{\prime}} \cdot \overrightarrow{\mathbf{n}}^{\prime}=0, \overrightarrow{\mathbf{m}}^{\prime} \cdot \overrightarrow{\mathbf{n}}^{\prime}=0,\left|\overrightarrow{\mathbf{m}}^{\prime}\right|=\left|\overrightarrow{\mathbf{n}}^{\prime}\right|=1$

$\tilde{\rho}, \tilde{u}, \tilde{v}, \tilde{w}$ are Roe averaged variables.

The second order central differencing is used for viscous flux $R_{i+1 / 2}$ on the left hand side of Eq. (30), and the Jacobian matrix is obtained as:

$\frac{\partial R_{i+1 / 2}}{\partial q_{i+1 / 2}} \delta q_{i+1 / 2}=\frac{\partial R_{i+1 / 2}}{\partial q_{i}} \delta q_{i}+\frac{\partial R_{i+1 / 2}}{\partial q_{i+1}} \delta q_{i+1}$

The final linearized equations of Eq. (30) can be written as the following

$$
\begin{aligned}
\bar{B} \Delta q_{i, j, k}^{n+1} & +A^{+} \Delta q_{i+1, j, k}^{n+1}+A^{-} \Delta q_{i-1, j, k}^{n+1}+B^{+} \Delta q_{i, j+1, k}^{n+1}+B^{-} \Delta q_{i, j-1, k}^{n+1} \\
& +C^{+} \Delta q_{i, j, k+1}^{n+1}+C^{-} \Delta q_{i, j, k-1}^{n+1}=R H S^{n}
\end{aligned}
$$

where

$\bar{B}=\Gamma+A+B+C$ 
and

$A=\frac{\Delta t}{J}\left[\left(\widehat{A}_{i+1 / 2}^{L}-L_{i+1 / 2}^{L}\right)-\left(\widehat{A}_{i-1 / 2}^{R}-L_{i-1 / 2}^{R}\right)\right]$

$A^{+}=\frac{\Delta t}{J}\left(\widehat{A}_{i+1 / 2}^{R}-L_{i+1 / 2}^{R}\right), \quad A^{-}=-\frac{\Delta t}{J}\left(\widehat{A}_{i-1 / 2}^{L}-L_{i-1 / 2}^{L}\right)$

$\widehat{A}_{i+1 / 2}^{R}=\frac{1}{2}\left(A_{i+1}-\widetilde{A}_{i+1 / 2}\right), \quad \widehat{A}_{i+1 / 2}^{L}=\frac{1}{2}\left(A_{i}+\widetilde{A}_{i+1 / 2}\right)$

$A_{i}=\left.\frac{\partial E}{\partial q}\right|_{i}, \quad \tilde{A}_{i+1 / 2}=\left.\left(\Gamma M_{\Gamma}\left|\Lambda_{\Gamma}\right| M_{\Gamma}^{-1}\right)\right|_{i+1 / 2}$

$L_{i+1 / 2}^{L}=\frac{\partial R_{i+1 / 2}}{\partial q_{i}}, \quad L_{i+1 / 2}^{R}=\frac{\partial R_{i+1 / 2}}{\partial q_{i+1}}$

$J$ is the transformation Jacobian. $B^{ \pm}, B$ and $C^{ \pm}, C$ have the same forms as $A^{ \pm}, A$. And

$$
\begin{aligned}
R H S^{n}= & \frac{\Delta t}{J}\left[\left(R_{i+1 / 2}^{n}-R_{i-1 / 2}^{n}\right)+\left(S_{j+1 / 2}^{n}-S_{j-1 / 2}^{n}\right)+\left(T_{k+1 / 2}^{n}-T_{k-1 / 2}^{n}\right)\right. \\
& \left.-\left(E_{i+1 / 2}^{n}-E_{i-1 / 2}^{n}\right)-\left(F_{j+1 / 2}^{n}-F_{j-1 / 2}^{n}\right)-\left(G_{k+1 / 2}^{n}-G_{k-1 / 2}^{n}\right)\right]
\end{aligned}
$$

Previous studies (for example [46-48]) have shown that the Gauss-Seidel line iteration provide a robust computational frame for implementing high order schemes. In a certain sweep direction, for example, in $\xi$ direction assuming the sweeping from small index value to large one, the Gauss-Seidel line iteration can be written as

$B^{-} \Delta q_{i, j-1, k}^{n+1}+\bar{B} \Delta q_{i, j, k}^{n+1}+B^{+} \Delta q_{i, j+1, k}^{n+1}=R H S^{\prime}$

where

$R H S^{\prime}=R H S^{n}-A^{+} \Delta q_{i+1, j, k}^{n}-A^{-} \Delta q_{i-1, j, k}^{n+1}-C^{+} \Delta q_{i, j, k+1}^{n}-C^{-} \Delta q_{i, j, k-1}^{n+1}$

\section{Results and discussion}

To demonstrate the effectiveness of the preconditioned LDE scheme, the test cases include a lid driven cavity, a cavity natural convection flow, a subsonic flat plate turbulent boundary layer, inviscid transonic converging-diverging nozzle flow, the transonic flow over RAE2822 airfoil, and the laminar wall boundary layer with Mach number ranging from $10^{-3}$ to 2.0.

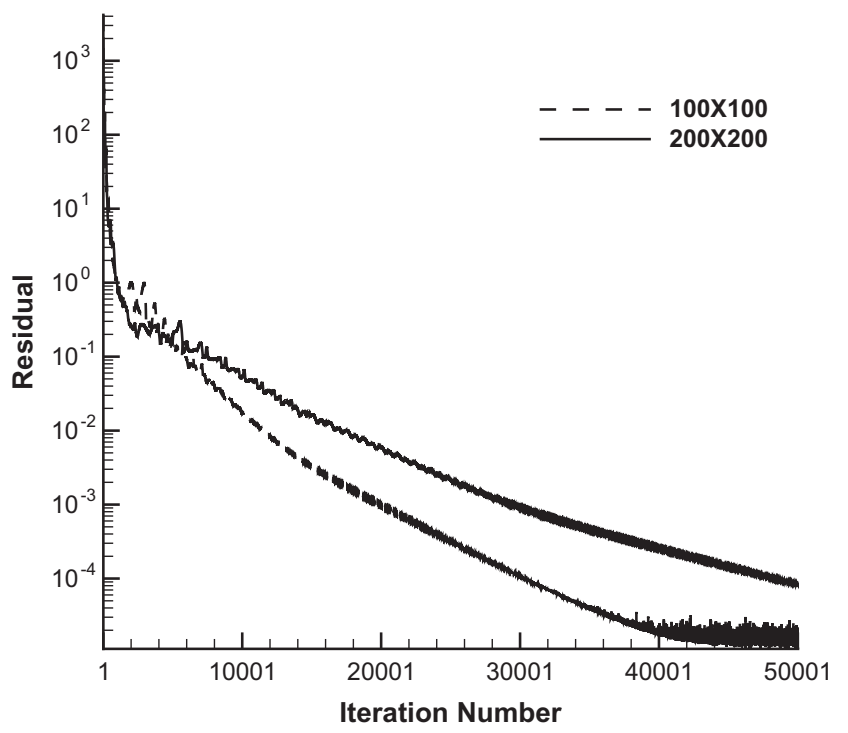

Fig. 1. Convergence rate of the lid-driven cavity flow, $R e=3200, M=10^{-3}$.
In this paper, at boundaries, the conservative variables are first obtained using various required boundary conditions [43], and then the primitive variables used in preconditioning system are calculated from the conservation variables accordingly.

The residual to measure the convergence history is defined as

$$
\begin{aligned}
\text { Residual } & =\operatorname{Max}\left\{\left|R H S^{n}\right|\right\}_{i, j, k} \\
& =\operatorname{Max}\left(\sqrt{R H S^{2}(1)+R H S^{2}(2)+\cdots+R H S^{2}(5)}\right)_{i, j, k}
\end{aligned}
$$

and $R H S^{n}$ is determined by Eq. (39).

\subsection{Lid driven cavity flow}

The lid driven cavity flow, which is a flow in a cavity with the lid moving at a constant speed, is a benchmark solution used to validate incompressible flow calculation $[31,49,50]$.

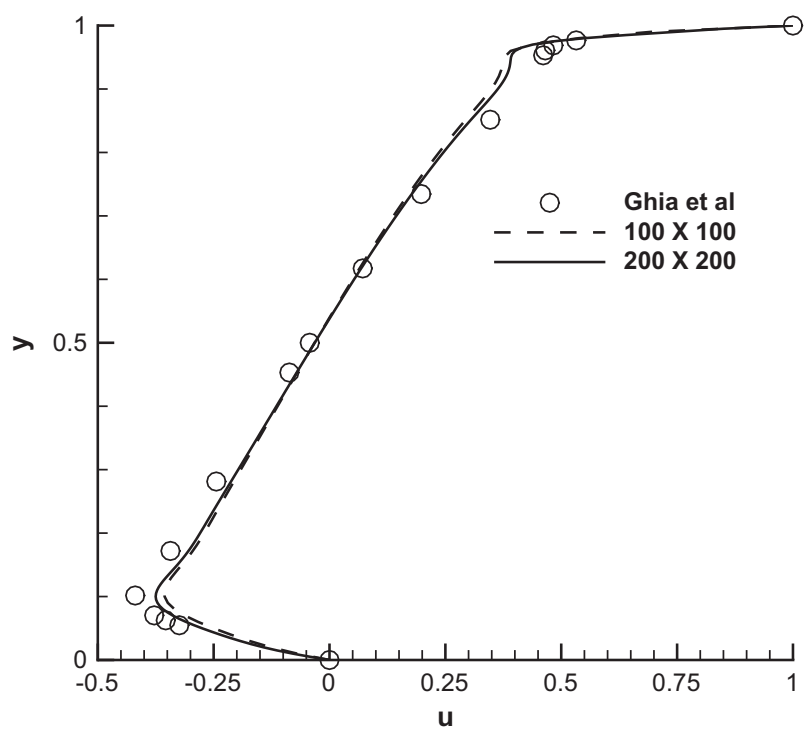

Fig. 2. Velocity $\mathrm{u}$ along the vertical centerline for the lid driven cavity flow, $R e=3200, M=10^{-3}$

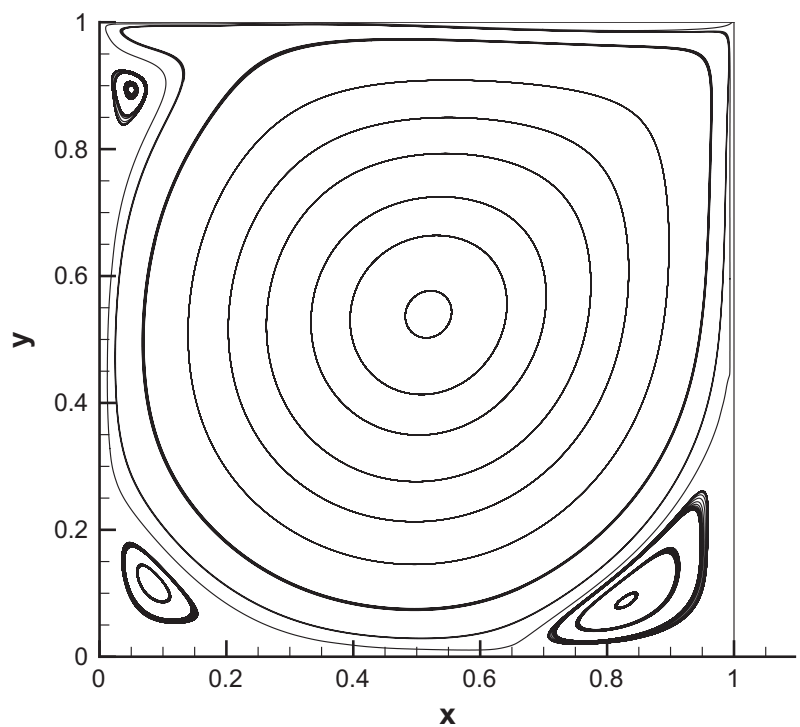

Fig. 3. Streamlines for the lid driven cavity flow, $100 \times 100$ 
The flowfield with Reynolds of 3200, no-slip isothermal wall boundary condition and a Mach number of $10^{-3}$ for the moving lid is calculated. For the purpose of comparison, the uniform mesh systems of $100 \times 100$ and $200 \times 200$ are used.

Fig. 1 gives the convergence histories and shows that the residual is reduced by $8-9$ order of magnitude. Since the primitive pressure $p$ is used in the present preconditioned method, the decreased Mach number results in increased machine round off errors, which increase proportionally with $M^{2}[32,23]$ and make the residual floating at the level of $10^{-5}$. It needs to point out that the method without preconditioning cannot get the correct solution for this case due to excessive dissipation.

Fig. 2 is the comparison of the velocity component in $x$-direction along the vertical centerline. The present results are in good agreement with that obtained by solving incompressible NavierStokes equations [49].

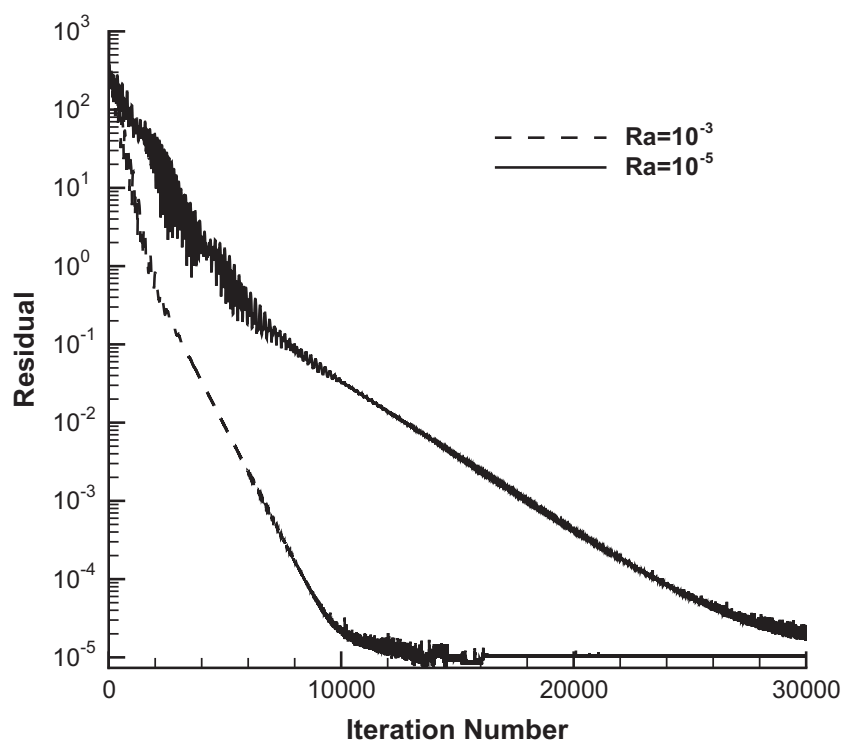

Fig. 4. Convergence rate of the cavity natural convection flows.

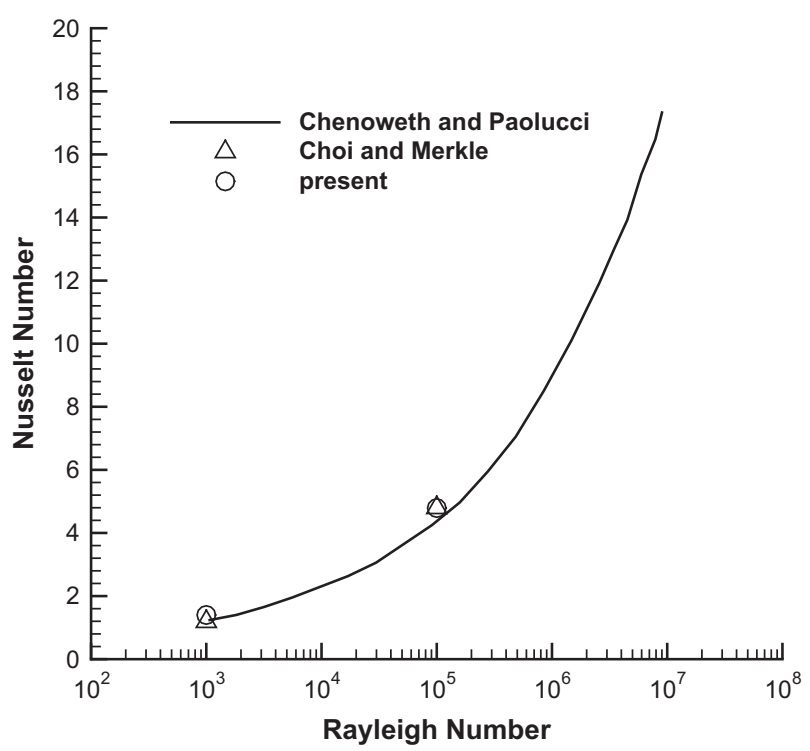

Fig. 5. Comparison of Nusselt number of the cavity natural convection flow.
Fig. 3 shows the streamlines calculated by the present method with the mesh of $100 \times 100$. It exhibits a large primary vortex with two secondary vortices in the two bottom corners and a secondary vortex near the upper-left corner, which is the same as other researchers predict $[31,49,50]$.

\subsection{Cavity natural convection flow}

The second test case is a cavity natural convection flow induced by a temperature difference of four times on the two vertical walls. This flow has very low velocity and is in the incompressible flow regime. The configuration consists of two insulated horizontal walls and two vertical walls at temperature $T_{h}$ and $T_{c}$ with $T_{h}=4 T_{c}$. In this paper, the natural convective flows at two Rayleigh number, $R a=10^{3}$ and $R a=10^{5}$, are calculated. The uniform mesh with size of $100 \times 100$ is used.

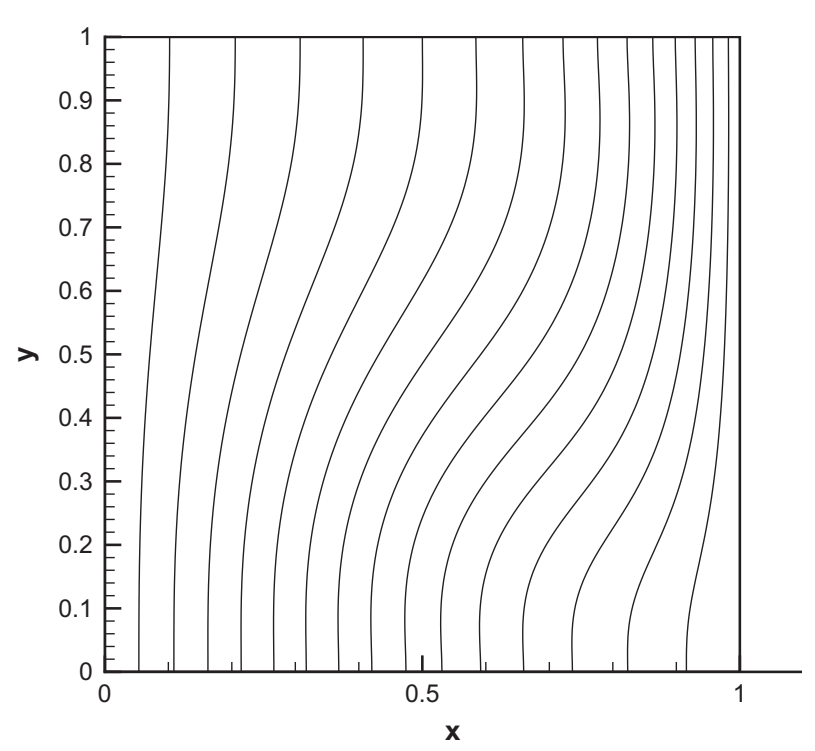

Fig. 6. Isoline temperature of the cavity natural convection flow, $R a=10^{3}$.

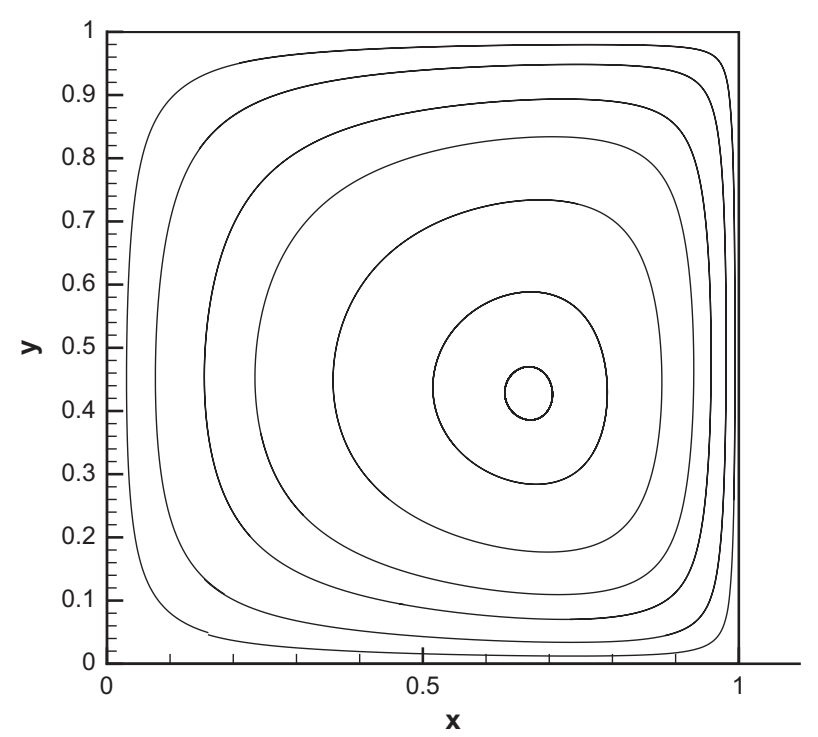

Fig. 7. Streamline of the cavity natural convection flow, $R a=10^{3}$. 
The convergence histories are shown in Fig. 4. Fig. 5 compares the Nusselt number at the left side wall with the result of Chenoweth and Paolucci [51] and Choi and Merkle [23], they are in excellent agreement. Figs. 6-9 show the streamline and temperature isolines. Figs. 7 and 9 indicate that the flow evolves from one vortex core to two vortex cores when the Rayleigh number is increased from $10^{3}$ to $10^{5}$. This is due to the instability induced by high Rayleigh number, and is consistent with the solutions obtained by other researchers [23]. Same as in the first test case, without preconditioning, the correct solution cannot be obtained.

\subsection{Wall boundary layer}

The third validation case is a steady state laminar boundary layer flow on an adiabatic flat plate to test the methodology for both compressible and incompressible flows. The Reynolds number based on the length of the flat plate is $4.0 \times 10^{4}$. The Prandtl

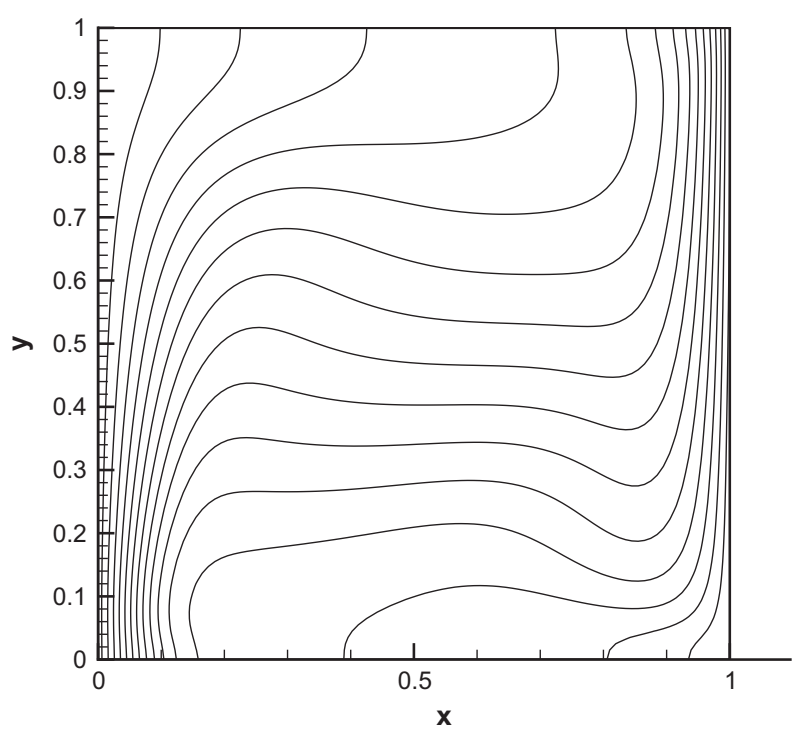

Fig. 8. Isoline temperature of the cavity natural convection flow, $R a=10^{5}$. number of 1.0 is used in order to compare with the analytical solution. The computation domain is taken to be $[0,2] \times[0,1.6]$. The mesh size is $180 \times 80$. Three cases with different incoming Mach number $\left(M_{\infty}=2.0, M_{\infty}=10^{-2}\right.$, and $\left.M_{\infty}=10^{-3}\right)$ are calculated:

Fig. 10 is the convergence histories of the case with Mach number of 2.0, the supersonic flat plate laminar flow, with and without preconditioning. It shows that the precondition method has the

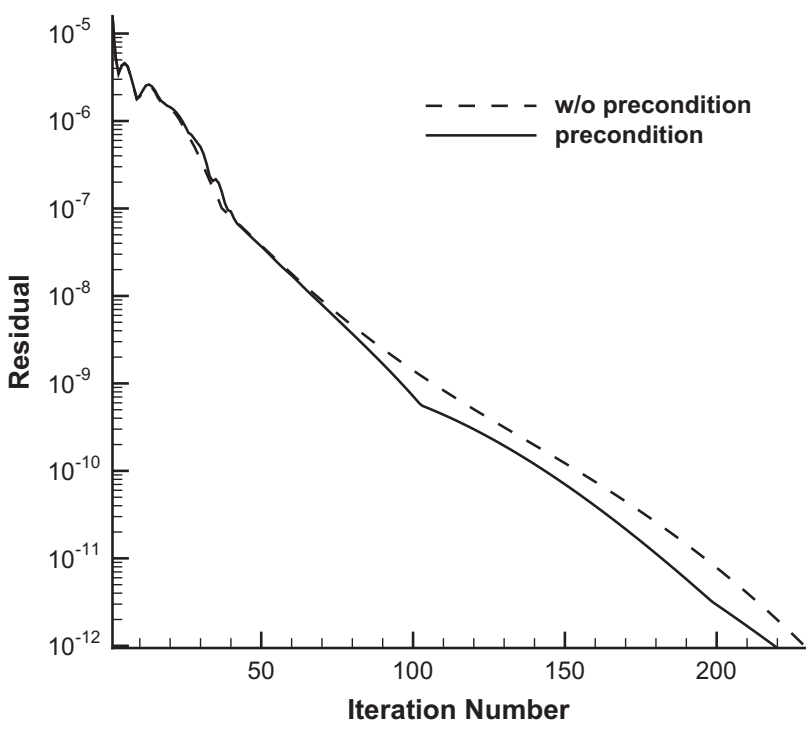

Fig. 10. Convergence rate of the supersonic boundary layer flow, $M=2.0$.

Table 4

The comparison of iteration number and CPU time.

\begin{tabular}{lccccc}
\hline Case & \multicolumn{2}{l}{ w/o Precondition } & & Precondition & \\
\cline { 2 - 3 } & $\begin{array}{l}\text { Iteration } \\
\text { number }\end{array}$ & CPU time & & $\begin{array}{l}\text { Iteration } \\
\text { number }\end{array}$ & CPU time \\
& 229 & $0.1012 \mathrm{e}+3$ & 220 & $0.1082 \mathrm{e}+3$ \\
$\begin{array}{l}\text { Supersonic } \\
\text { plate }\end{array}$ & 17,302 & $0.3207 \mathrm{e}+4$ & 6539 & $0.1286 \mathrm{e}+4$ \\
$\begin{array}{l}\text { Nozzle } \\
\text { RAE-2822 }\end{array}$ & 17,182 & $0.4062 \mathrm{e}+4$ & 10354 & $0.2753 \mathrm{e}+4$ \\
\hline
\end{tabular}

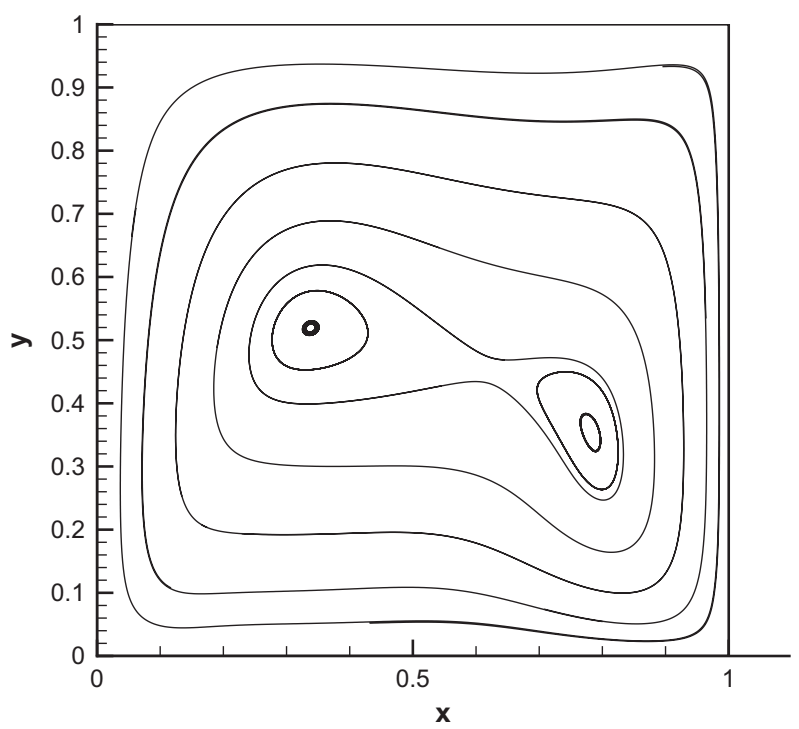

Fig. 9. Streamline of the cavity natural convection flow, $R a=10^{5}$.

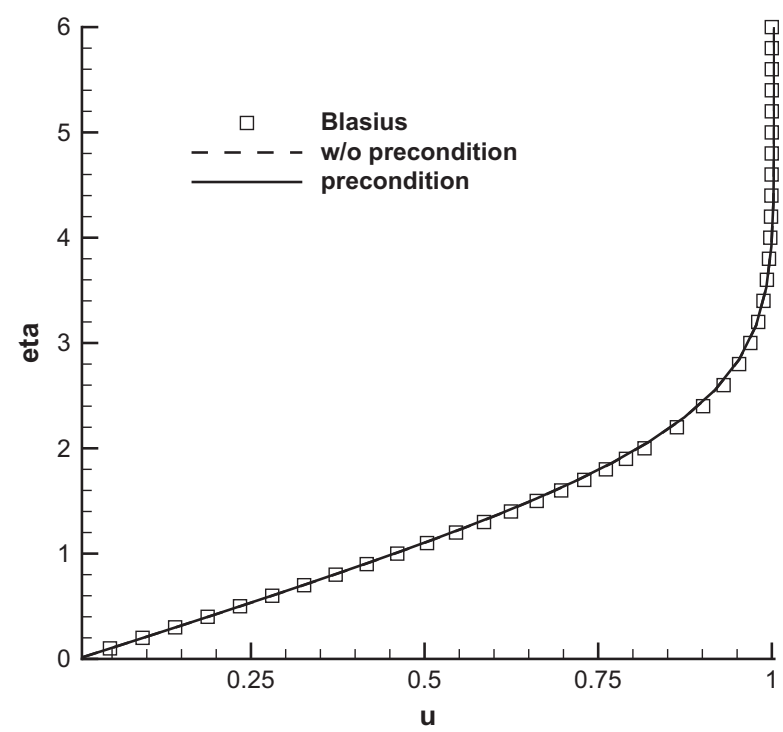

Fig. 11. Velocity profile of the supersonic boundary layer flow, $M=2.0$ 
same convergence rate as the one without preconditioning. For this supersonic flow, the thin subsonic boundary layer has created little stiffness and hence the advantage of preconditioning is not significant. From the comparison of CPU time in Table 4, it is found that the preconditioning method used a little bit more than the one without preconditioning.

The computed velocity and temperature profiles of this supersonic laminar boundary layer shown in Figs. 11 and 12 indicate that the numerical results agree excellently with the Blasius solution.

Figs. 13 and 14 show the convergence histories of the cases with $M=10^{-2}$ and $M=10^{-3}$. It can be seen that the residual without preconditioning oscillate at a high level with a large amplitude. For the cases with $M_{\infty}=10^{-2}$, the residual with preconditioning is about six orders of magnitude lower than the one without preconditioning. For the case with $M_{\infty}=10^{-3}$, the residual with preconditioning is reduced to the level of machine zero, which is higher than the $M=2.0$ case due to increased pressure level.

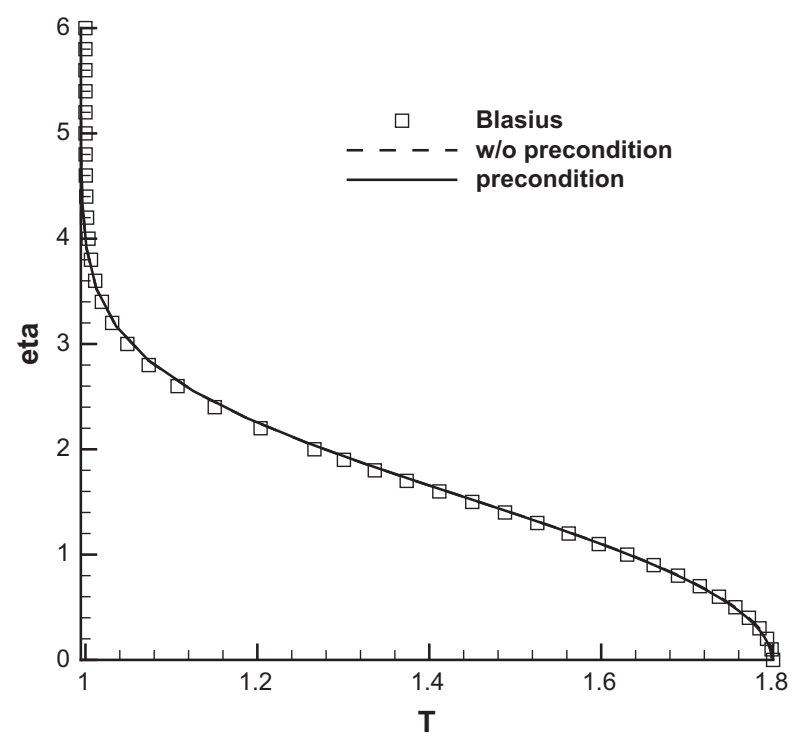

Fig. 12. Temperature profile of the supersonic boundary layer flow, $M=2.0$.

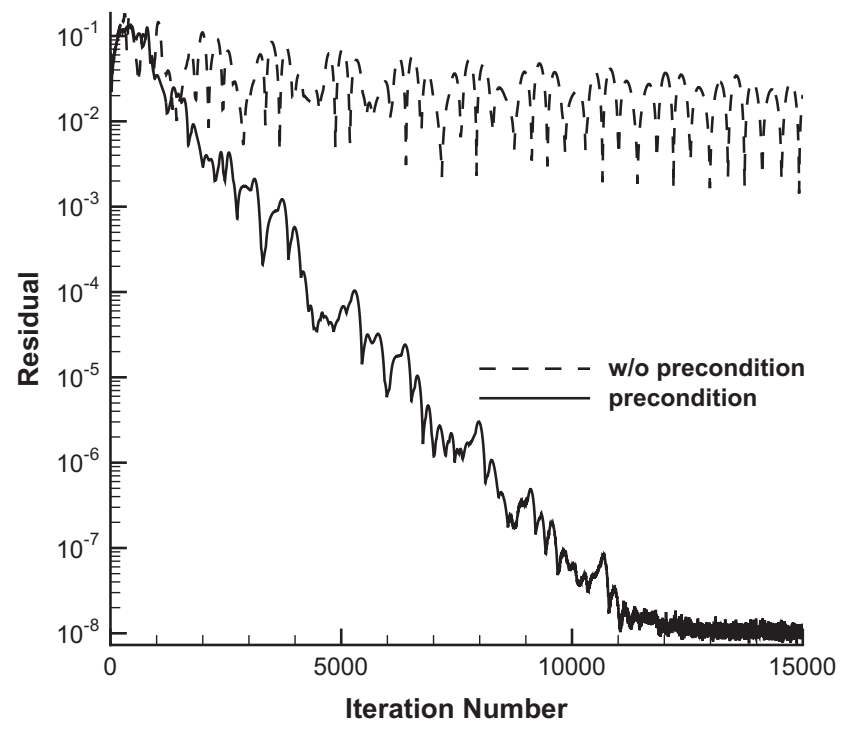

Fig. 13. Convergence rate of the subsonic boundary layer flow, $M=10^{-2}$.
Figs. 15 and 16 are the comparison of numerical results with Blasius solution for $M=10^{-2}$ and $10^{-3}$. Fig. 15 shows that, even though its solution is less accurate than the preconditioned one, the flow solver without preconditioning can still resolve the boundary layer at $M=10^{-2}$. However, the velocity profile in Fig. 16 demonstrates that, for the case with $M=10^{-3}$, the numerical solution without preconditioning is significantly diffused due to the large numerical dissipation, whereas the preconditioned solvers accurately resolve the velocity profile.

\subsection{Transonic converging-diverging nozzle}

To examine the performance of the preconditioning methodology in two-dimensional flow and the capability to capture shock waves, an inviscid transonic converging-diverging nozzle is calculated. The nozzle was designed and tested at NASA and was named as nozzle A1 [52]. Due to the geometric symmetry about the center line, only the upper half of the nozzle is calculated. The mesh size is

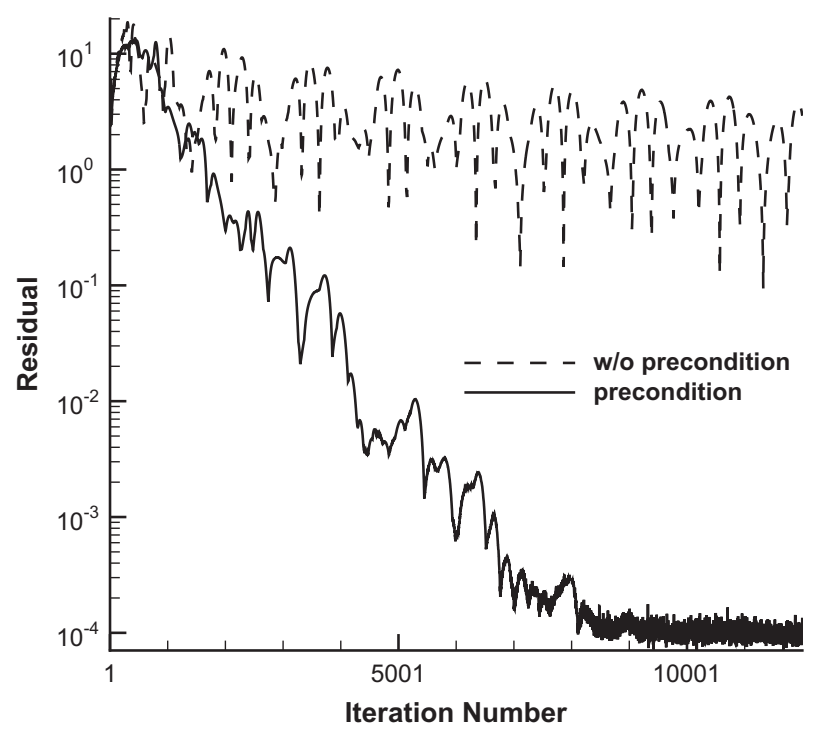

Fig. 14. Convergence rate of the subsonic boundary layer flow, $M=10^{-3}$.

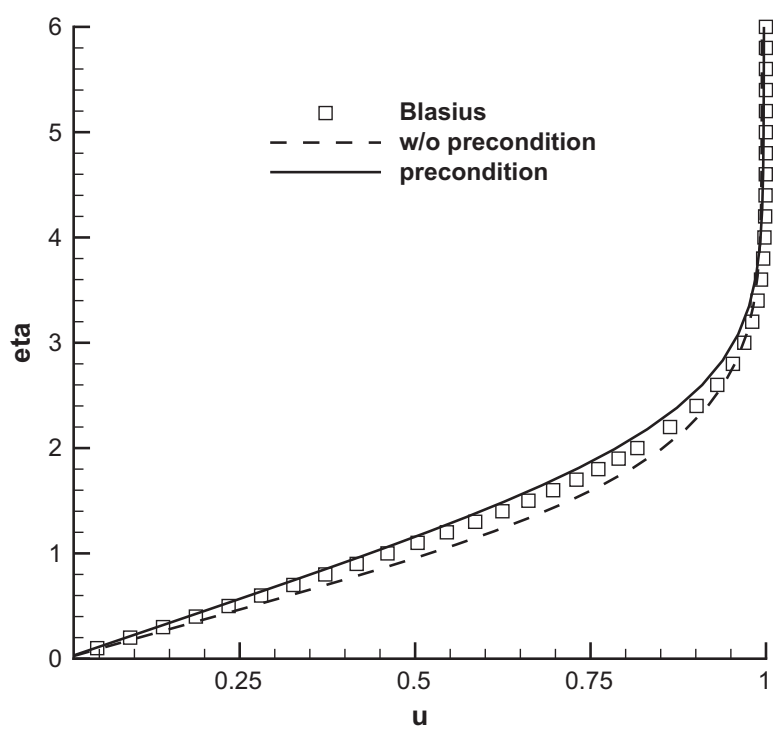

Fig. 15. Velocity profile of the subsonic boundary layer flow, $M=10^{-2}$. 
$175 \times 50$. The grid is clustered near the wall. The inlet Mach number is 0.28 .

Fig. 17 shows the Mach contours obtained by present preconditioning method. Fig. 18 is the comparison of the convergence his-

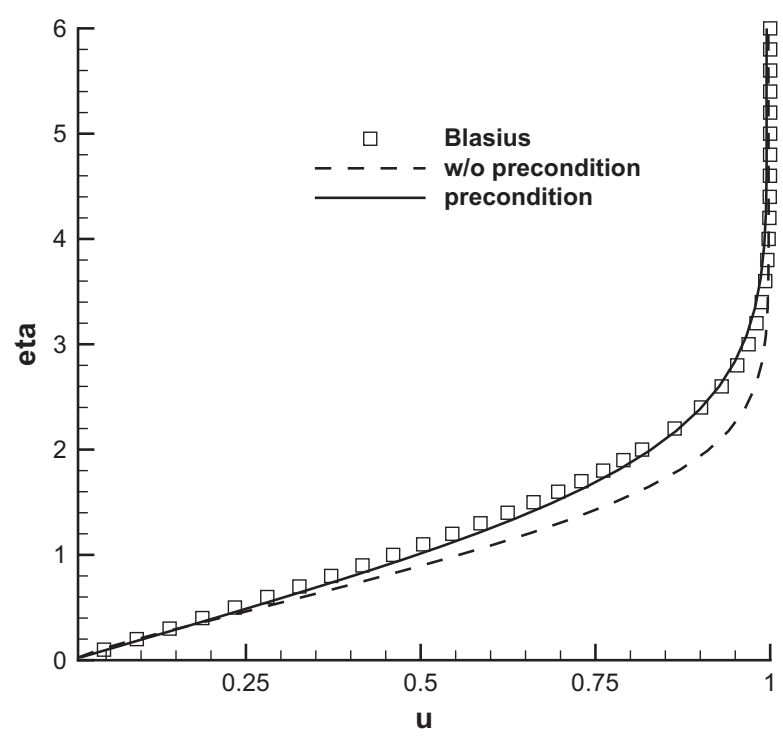

Fig. 16. Velocity profile of the subsonic boundary layer flow, $M=10^{-3}$.

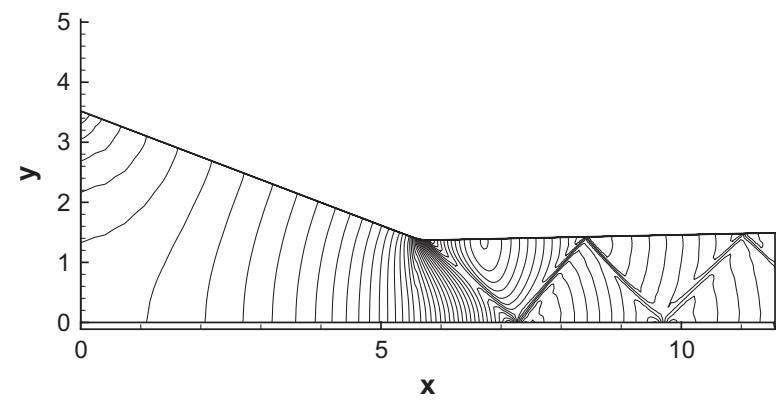

Fig. 17. Mach number contours of the transonic converging-diverging nozzle flow.

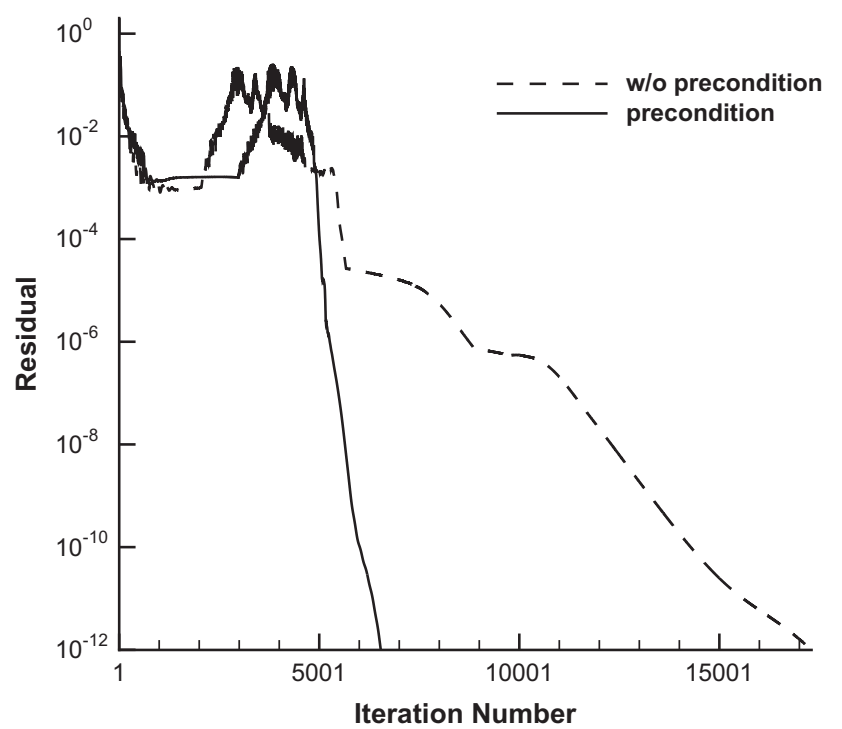

Fig. 18. Convergence rate of the transonic converging-diverging nozzle flow. tories with and without precondition. The preconditioned convergence rate is about $60 \%$ faster than the one without preconditioning, and the CPU time is only $40 \%$ of the latter, see Table 4 .

Fig. 19 shows the pressure coefficients at the upper wall surface. It can be seen that both the methods with and without preconditioning obtain identical results.

\subsection{Transonic RAE2822 airfoil}

To further examine the preconditioning method for transonic compressible flows, the steady state solution of the transonic RAE2822 airfoil is calculated using the Reynolds averaged Navier-Stokes equations with the Baldwin-Lomax turbulence model. The mesh size is $256 \times 55$, the freestream Mach number $M_{\infty}$ is 0.729 , the Reynolds number based on chord is $6.5 \times 10^{6}$, and the angle of attack is $2.31^{\circ}$.

From Fig. 20, we can see that the preconditioning method only needs about $60 \%$ of the iteration numbers of the one without

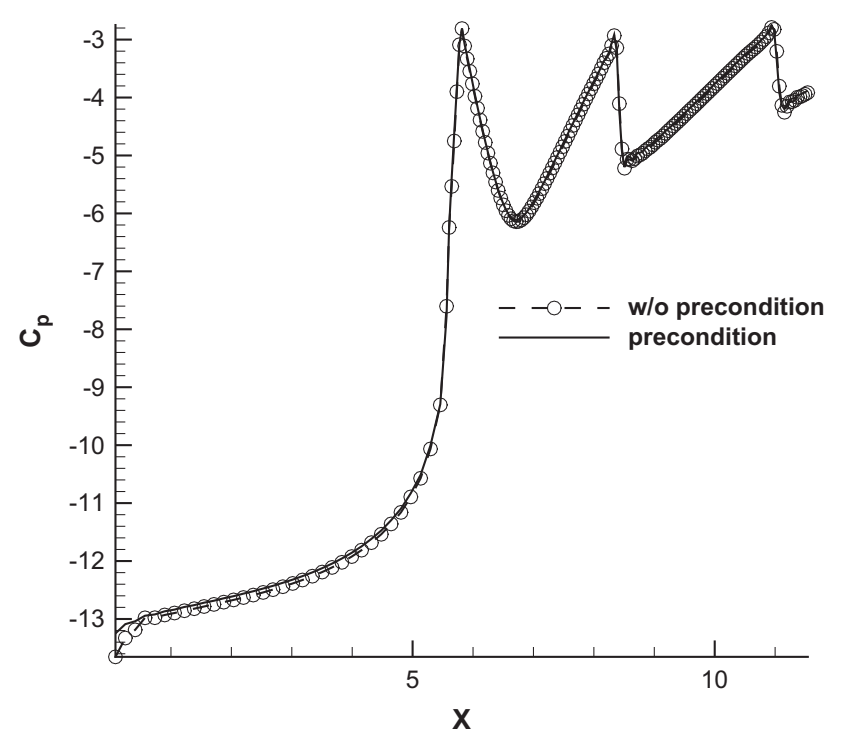

Fig. 19. The pressure coefficients at the upper wall of the transonic convergingdiverging nozzle flow.

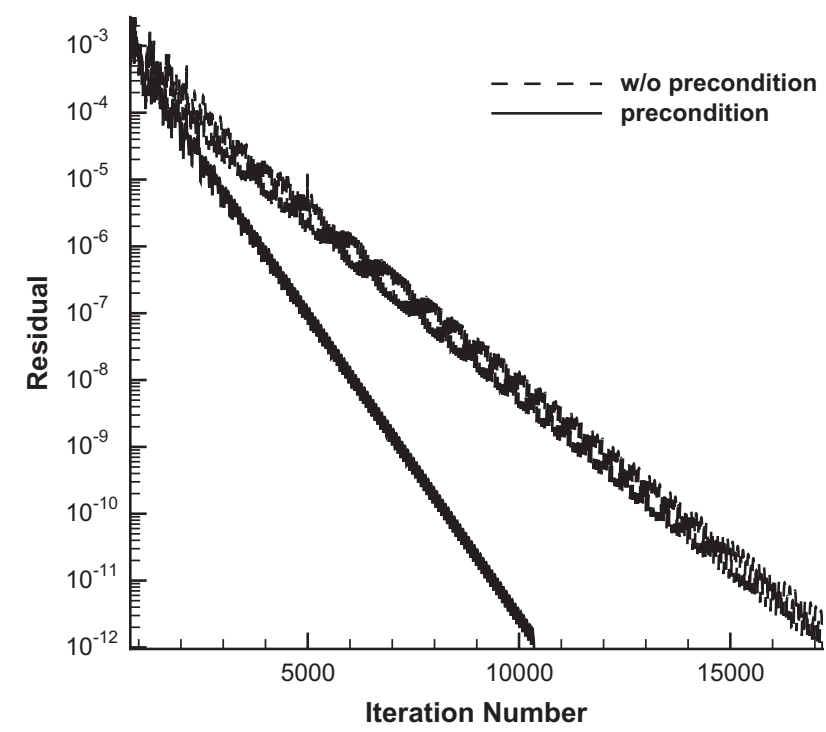

Fig. 20. Convergence rate of the transonic flow over RAE2822 airfoil. 


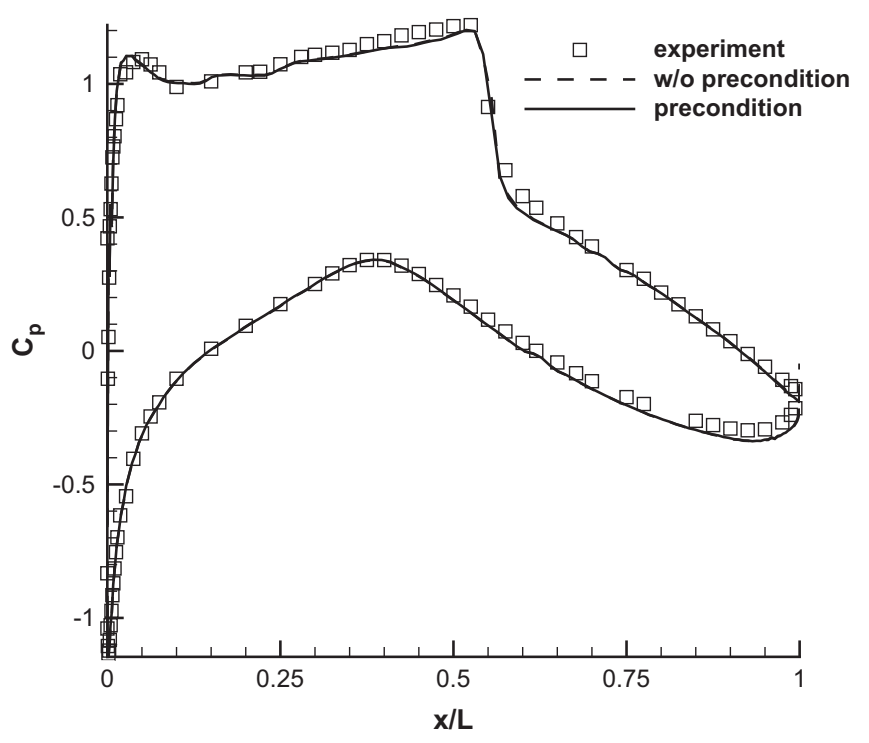

Fig. 21. The pressure coefficients at the airfoil surface of the transonic flow over RAE2822 airfoil.

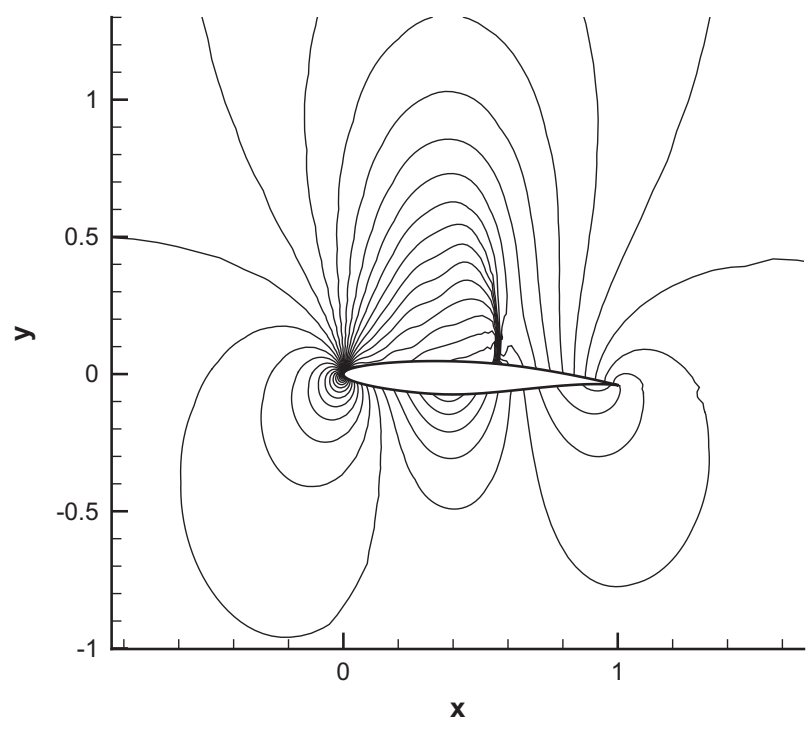

Fig. 22. Pressure contours of the transonic flow over RAE2822 airfoil

preconditioning to converge to machine zero, and the CPU time is only $67 \%$ of the latter (4).

Fig. 21 is the comparisons of the coefficients of pressure on the wall. They show that both the results with and without preconditioning are identical and are in excellent agreement with the experiment. Fig. 22 shows the pressure contours obtained by the present preconditioning method.

\section{Conclusions}

A low diffusion E-CUSP (LDE) scheme is developed for preconditioned Navier-Stokes equations. Different from H-CUSP schemes, the pressure term is separated from the total enthalpy in the energy equation and is also preconditioned in the E-CUSP scheme. Combined with the 5th-order WENO scheme for inviscid flux, the high order difference scheme for viscous terms, and the unfactored implicit Gauss-Seidel relaxation scheme for time integration, in which the preconditioned Roe's matrices are used, the algorithm is used to calculate flow fields from very low speed incompressible flows to supersonic compressible flows.

The numerical simulation of lid-driven and natural convective incompressible cavity flows, low subsonic incompressible flows, transonic and supersonic compressible flows show that the preconditioning method is efficient, accurate and robust, not only for the low Mach number incompressible flows, but also for the subsonic and transonic compressible flows. For high subsonic and transonic flows, the preconditioning also accelerates convergence due to reduced stiffness in near wall low speed region. For low speed incompressible flows, the preconditioning is necessary not only to remove the stiffness, but also to reduce numerical dissipation to ensure accurate results.

\section{Acknowledgment}

This work is supported by Miami Wind ${ }^{\mathrm{TM}}$ Research Center at University of Miami.

\section{References}

[1] Jameson A. Analysis and design of numerical schemes for gas dynamics I: Artificial diffusion, upwind biasing, limiters and their effect on accuracy and multigrid convergence in transonic and hypersonic flow. AIAA paper 93-3359; July, 1993.

[2] Jameson A. Analysis and design of numerical schemes for gas dynamics I: Artificial diffusion, upwind biasing, limiters and their effect on accuracy and multigrid convergence in transonic and hypersonic Flow. J Comput Fluid Dynam 1995;4:171-218.

[3] Jameson A. Analysis and design of numerical schemes for gas dynamics II: Artificial diffusion and discrete shock structure. J Comput Fluid Dynam 1995;5:1-38.

[4] Liou M-S, Steffen CJ. A new flux splitting scheme. J Comput Phys 1993;107:23-39.

[5] Wada Y, Liou M-S. An accurate and robust splitting scheme for shock and contact discontinuities. AIAA paper 94-0083; 1994.

[6] Liou M-S. Progress towards an improved CFD methods: AUSM+. AIAA Paper 951701-CP; June, 1995

[7] Liou M-S. A sequel to AUSM: AUSM+ ${ }^{+}$J Comput Phys 1996;129:364-82.

[8] Liou M-S. Ten years in the making - AUSM-family. AIAA 2001-2521; 2001.

[9] Kim Kyu Hong, Kim Chongam, Rho Oh-Hyun. Methods for the accurate computations of hypersonic flows: I. AUSMPW+Scheme. J Comput Phys 2001;174:38-80.

[10] Hänel D, Schwane R, Seider G. On the accuracy of upwind schemes for the Solution of the Navier-Stokes eqautions. AIAA paper 87-1105 CP; 1987.

[11] Edwards JR. A low-diffusion flux-splitting scheme for Navier-Stokes calculations. AIAA paper 95-1703-CP; June, 1995.

[12] Edwards JR. A low-diffusion flux-splitting scheme for Navier-Stokes calculations. Comput Fluids 1997;6:635-59.

[13] Zha G-C, Bilgen E. Numerical solutions of Euler equations by using a new flux vector splitting scheme. Int J Numer Methods Fluids 1993;17:115-44.

[14] Zha G-C. Numerical tests of upwind scheme performance for entropy condition. AIAA J 1999;37:1005-7.

[15] Zha G-C. Comparative study of upwind scheme performance for entropy condition and discontinuities. AIAA Paper 99-CP-3348; June 28-July 1, 1999.

[16] Zha G-C, Hu Z-J. Calculation of transonic internal flows using an efficient high resolution upwind scheme. AIAA J 2004;42(2):205-14.

[17] Zha GC, Shen YQ, Wang BY. An improved low diffusion E-CUSP upwind scheme. Comput Fluids 2011;48:214-20.

[18] Zha G-C. A low diffusion efficient upwind scheme. AIAA J 2005;43:1137-40.

[19] Zha G-C. A low diffusion E-CUSP upwind scheme for transonic flows. In: 34th AIAA fluid dynamics conference. AIAA paper 2004-2707, to appear in AIAA Journal; June 28-July 1, 2004.

[20] Turkel E. Preconditioned methods for solving the incompressible and low speed compressible equations. J Comput Phys 1987;72:277-98.

[21] Merkle CL, Choi YH. Computation of low-speed compressible flows with timemarching procedures. Int J Numer Methods Eng 1988;25:293-311.

[22] Turkel E. Preconditioning techniques in computational fluid dynamics. Ann Rev Fluid Mech 1999;31:385-416.

[23] Choi YH, Merkle CL. The application of preconditioning in viscous flows. J Comput Phys 1993;105:207-23.

[24] Weiss JM, Smith WA. Preconditioning applied to variable and constant density flows. AIAA J 1995;33:2050-7.

[25] Thornber B, Drikakis D, Williams R, Youngs D. On entropy generation and dissipation of kinetic energy in high-resolution shock-capturing schemes. J Comput Phys 2008;227:4853-72.

[26] Thornber B, Mosedale A, Drikakis D, Youngs D, Williams R. An improved reconstruction method for compressible flows with low Mach number features. J Comput Phys 2008;227:4873-94. 
[27] Merkle CL, Sullivan JY, Buelow PEO, Venkateswaran S. Computation of flows with arbitrary equations of state. AIAA J 1998;36:515-21.

[28] De Bortoli AL. Multigrid based aerodynamical simulations for the NACA 0012 airfoil. Appl Numer Math 2002;40:337-49.

[29] Weiss JM, Maruszewski JP, Smith WA. Implicit solution of preconditioned Navier-Stokes equations using algebraic multigrid. AIAA J 1999;37:29-36.

[30] Rossow C-C. Efficient computation of compressible and incompressible flows. J Comput Phys 2007;220:879-99.

[31] Huang J-C, Lin H, Yang J-Y. Implicit preconditioned WENO scheme for steady viscous flow computation. J Comput Phys 2009;228:420-38.

[32] Shen Y-Q, Zha G-C. Simulation of flows at all speeds with high-order WENO schemes and preconditioning. In: 47th AIAA aerospace sciences meeting, AIAA-2009-1312, January 2009.

[33] Briley WR, Taylor LK, Whitfield DL. High-resolution viscous flow simulations at arbitrary Mach number. J Comput Phys 2003;184:79-105.

[34] Turkel E, Radespiel R, Kroll N. Assessment of preconditioning methods for multidimensional aerodynamics. Comput Fluids 1997;26:613-34.

[35] Patel G, Drikakis D. Effects of preconditioning on the accuracy and efficiency of incompressible flows. Int J Numer Methods Fluids 2005;47:963-70.

[36] Edwards JR, Liou M-S. Low-diffusion flux-splitting methods for flows at all speeds. AIAA J 1998;36:1610-7.

[37] Edwards J, Franklin R, Liou M-S. Low diffusion flux-splitting methods for real fluid flows with phase transitions. AIAA J 2000;38:1624-33.

[38] Mao D, Edwards JR, Kuznetsov AV, Srivastava RK. Development of lowdiffusion flux-splitting methods for dense gas-solid flows. J Comput Phys 2003;185:100-19.

[39] Neaves MD, Edwards JR. All-speed time-accurate underwater projectile calculations using a preconditioning algorithm. J Fluids Eng 2006;128:284-96.

[40] Liou MS. A sequel to AUSM, Part II: AUSM+ -up for all speeds. J Comput Phys 2006;214:137-70.
[41] Ihm SW, Kim C. Computations of homogeneous-equilibrium two-phase flows with accurate and efficient shock-stable schemes. AIAA J 2008;46:3012-37.

[42] van Leer B. Towards the ultimate conservative difference scheme, III. J Comput Phys 1977;23:263-75.

[43] Shen Y-Q, Zha G-C, Wang B-Y. Improvement of stability and accuracy of implicit WENO scheme. AIAA J 2009;47:331-44.

[44] Jiang G-S, Shu C-W. Efficient implementation of weighted ENO schemes. J Comput Phys 1996;126:202-28.

[45] Shen Y-Q, Zha G-Z, Chen X-Y. High order conservative differencing for viscous terms and the application to vortex-induced vibration flows. J Comput Phys 2009;228:8283-300.

[46] Shen Y-Q, Wang B-Y, Zha G-C. Comparison study of implicit Gauss-Seidel line iteration method for transonic flows. AIAA-2007-4332, June 2007.

[47] Drikakis D, Tsangaris S. On the solution of the compressible Navier-Stokes equations using improved flux vector splitting methods. Appl Math Modell 1993;17:282-97.

[48] Bagabir A, Drikakis D. Numerical experiments using high-resolution schemes for unsteady, inviscid, compressible flows. Comput Methods Appl Mech Eng 2004;193:4675-705.

[49] Ghia U, Ghia KN, Shin CT. High-Re solutions for incompressible flow using the Navier-Stokes equations and a multigrid method. J Comput Phys 1982;48:387-411.

[50] Yu Sheng-Tao, Jiang Bo-Nan, Liu Nan-Suey, Wu Jie. The least-squares finite element method for low-mach-number compressible viscous flows. Int J Numer Methods Eng 1995;38:3591-610.

[51] Chenoweth DR, Paolucci S. Natural convection in an enclosed vertical air layer with large horizontal temperature differences. J Fluid Mech 1986;169:173-210.

[52] Mason ML, Putnam LE. The effect of throat contouring on two-dimensional converging-diverging nozzles at static conditions. NASA technical paper 1704, 1980. 